Basic Health Sciences

Poster

Abstract ID: 25

\title{
Tualang honey improves non alcoholic steatohepatitis animal model
}

Azril Shahreez Abdul Ghani ${ }^{\mathrm{a}}$ | Nor Zamzila Abdullah ${ }^{\mathrm{b}}$ | Siti Aesah @ Naznin Muhammad ${ }^{\mathrm{b}}$ | Roslina Abdul Rahim $^{\mathrm{a}}$

${ }^{a}$ Department of Basic Medical Sciences, Kulliyyah of Medicine, International Islamic University Malaysia

${ }^{b}$ Department of Pathology and Laboratory Medicine, Kulliyyah of Medicine, International Islamic University Malaysia

Introduction: Non-alcoholic Steatohepatitis (NASH) is an emerging chronic liver disease with limited therapy available. Studies utilizing animal models induced with cholesterol diet ranging from 1-2\% are hampered by inconsistent yield of NASH features. Therefore, we aimed to establish a NASH animal model utilizing $12 \%$ cholesterol diet (CD) and to investigate the effects of Tualang honey (TH) known for its anti-inflammatory and antioxidative properties in this model. Methods: Twenty-four Sprague-Dawley rats were divided into 2 groups (12\% CD and standard diet) and were fed for 6 weeks. Following the establishment of $\mathrm{NASH}$, the rats in the $12 \% \mathrm{CD}$ group were subsequently divided into 3 groups. The first group was continued with only $12 \% \mathrm{CD}$. In the other 2 groups in addition to the $12 \% \mathrm{CD}$ they were given $\mathrm{TH}$ treatment at different concentrations (1.2 and 2.4 $\mathrm{g} / \mathrm{kg} /$ day) for 4 weeks. Blood biochemical analysis and histological assessment of liver were subsequently performed. Results: The liver histological sections of the rats fed with $12 \%$ CD showed macrovesicular steatosis, ballooning degeneration with lobular and portal inflammation. They also had increased serum alanine aminotransferase (ALT), total cholesterol (TC), low density lipoprotein cholesterol (LDL-C), fasting insulin, HOMA-IR and reduced high density lipoprotein cholesterol (HDL-C). Meanwhile, the TH treatment groups exhibited significant improvement in both the NASH grading and activity scores. The ALT, LDL-C, TC, triglyceride (TG), fasting insulin and HOMA-IR levels were reduced significantly. Conclusions: The $12 \%$ CD was able to induce NASH in the animal model. Tualang honey improved insulin sensitivity, dyslipidaemia, steatohepatitis.

KEYWORDS: NASH, cholesterol diet, Tualang Honey 